\title{
El modelo de Administración propuesto por la LOFAGE
}

Lourdes Máiz Carro *

\section{Necesidad de reformar la Administración General del Estado (AGE)}

\section{Reorganización administrativa y proceso autonómico}

La AGE ha resultado profundamente afectada por el nuevo modelo de Estado que la Constitución establece, y muy en particular por el título VIII, lo cual era lógico, puesto que hasta la aprobación de la Constitución llevaba el peso de la actuación del Estado y en 1980 absorbía el 89,95\% del gasto de las Administraciones Públicas.

No obstante, el impacto sobre la AGE de esta transformación constitucional, que ha conducido a acuñar el término Estado de las Autonomías, no se manifestó en toda su intensidad desde el primer momento, fundamentalmente por dos razones:

En primer lugar, porque a partir de los años ochenta se produce un fuerte crecimiento del gasto público, cuya razón fundamental es la necesidad de cubrir el retraso de España respecto de los paises de nuestro entomo en gasto social e infraestructuras. Ello provocó que la Administración del Estado transfiriera funciones, servicios y medios a las Comunidades Autónomas pero, al tiempo, viera aumentado el volumen de sus recursos. Es decir, iba disminuyendo su porcentaje en el gasto de las Administraciones Públicas, pero éste por su parte iba creciendo en términos absolutos.
En segundo lugar, porque el trasvase de funciones y servicios a las Comunidades Autónomas se va produciendo progresivamente a lo largo de los años, y esta gradualidad, contemplada por la Constitución, no exige de la Administración del Estado una transformación inmediata y total sino una acomodación progresiva.

El problema para que la organización y el funcionamiento de la AGE diera adecuada respuesta a la necesidad de su transformación estriba en que no se produjo esa acomodación gradual requerida, lo que provocó importantes desfases entre el modelo organizativo y los retos a los que la AGE debía hacer frente: desarrollar con eficacia las funciones que le siguen correspondiendo (gestión de importantes servicios públicos) y las nuevas que el propio Estado autonómico y la incorporación a la U.E. le confieren (planificación, coordinación con las CC.AA., colaboración con las Administraciones territoriales, formación de la voluntad estatal y su defensa en las instituciones comunitarias, etc.).

Una de las causas que explican, sin justificar, la insuficiente adecuación de la periferia, es la multiplicidad de fórmulas organizativas que sobre el territorio de las CC.AA.,desplegaron los Ministerios sectoriales, a pesar de los intentos normativos de ahormar los servicios territoriales de la AGE.

En efecto, ya en 1981 (pensemos que los primeros Estatutos de Autonomía se aprueban en diciembre de 1979) se publica un Real Decreto de reforma de la Administración periférica. Los dos rasgos básicos de este Decreto son, por una parte, el establecimiento de las direcciones provinciales como núcleo único de organización de los servicios provinciales de cada Ministerio y sus Organismos Autónomos. Las direcciones provinciales departamentales tendrán el carácter de unidades de gestión y ejecución de la política del Gobierno y de sus progra- 
mas de actuación correspondiente. (art. 5.1). En segundo lugar, se apunta la posibilidad de agrupar los servicios de varios Ministerios en una misma Dirección provincial, o agruparlos en Direcciones territoriales o regionales. Las Direcciones provinciales se situarían bajo la autoridad del Gobemador, y las territoriales bajo la autoridad del Delegado; pero dependerían orgánica y funcionalmente de sus respectivos Ministerios.

La Ley del Proceso Autonómico de $\mathbf{1 9 8 3}$ y el Real Decreto de Medidas de reorganización de la Administración periféri$\mathrm{ca}$, de ese mismo año, son mucho más estrictos y ordenan suprimir las Delegaciones ministeriales (salvo las de Hacienda) y reagrupar los servicios que deban subsistir bajo la autoridad del Gobernador Civil, único delegado de la Administración del Estado en las provincias, o del Delegado del Gobierno si tuvieran ámbito regional.

También, conforme a la Ley del Proceso Autonómico, los servicios centrales de los Ministerios y de sus organismos debían reorganizarse para acomodarlos a las funciones que, de acuerdo con el proceso autonómico, siguieran perteneciendo a los mismos, suprimiendo las estructuras de gestión que resultaran innecesarias y reconvirtiéndolas, en su caso, en servicios de coordinación, planificación, inspección, etc.

La rápida evolución normativa no se correspondia desde luego con la evolución de la realidad administrativa. Las normas anticipan un modelo que tardará catorce años en hacerse verdaderamente efectivo: su implantación es un empeño del actual Gobierno que empezará a hacerse realidad en los próximos meses, con el Real Decreto, ya en elaboración, de integración de servicios, reorganización de las Delegaciones y Subdelegaciones del Gobierno y supresión de las Direcciones provinciales o territoriales de los servicios integrados.

La Ley del Proceso Autonómico pensaba en una Administración del Estado reducida después de los traspasos, pero la realidad era que la AGE seguía disponiendo de un gran volumen de servicios periféricos, lo que hacía en la práctica inviable el modelo legal; no ya la dependencia orgánica de todos los servicios del Delegado o Gobernador, sino de las propias Direcciones provinciales ministeriales (que siguieron subsistiendo), tal y como propugnaba el Real Decreto de 1981.

La quiebra del modelo teórico dio lugar a que cada Ministerio estructurase sus servicios periféricos conforme a las circunstancias en presencia, con multiplicidad de orientaciones. Tampoco los servicios centrales aplicaron los criterios legalmente establecidos. En unos y otros quedaron unidades de gestión con escasas funciones según iban avanzando los traspasos, así como con unidades cuyos efectivos sobrepasaban en mucho las funciones asignadas. Al tiempo, muchos de los grandes servicios públicos estatales se encontraban con dificultades para reclutar su personal, por falta de medidas efectivas de redistribución de efectivos.

Por lo que atañe específicamente a la Administración periférica, la coordinación y dirección general de los servicios periféricos tampoco se consiguió con la presencia a nivel territorial de una figura constitucional, el Delegado del Gobierno, y unas figuras no constitucionales pero de gran raigambre histórica: los Gobernadores Civiles de perfil político, no dependientes jerárquicamente del Delegado del Gobierno, y que asumían una función tan importante como el mando de las fuerzas y cuerpos de seguridad.

La realidad es que la figura de los Gobernadores civiles se habia quedado superada tras la Constitución, tanto por el proceso de distribución territorial del poder político llevado a cabo como consecuencia del desarrollo del Estado autonómico, como por la consiguiente transformación de las Administraciones Públicas. En este contexto, dado que la Constitución no contempla a los Gobernadores civiles y sí a los Delegados del Gobierno, la desaparición antes o después de los Gobernadores era inevitable.

La dispersión y multiplicidad organizativa de los servicios periféricos antes aludida, condujo a que la dirección de los servicios estatales y la coordinación con los de las CC.AA., (art. 154 de la Constitución) fuera más un deseo que una realidad. No se evitaron las duplicidades e ineficiencias y, además, la insuficiente información del Delegado le impedía hacer propuestas a los Ministerios afectados o al Gobierno para solucionar tales disfunciones.

La AGE, en suma, desde la perspectiva de la organización y del funcionamiento diario de sus servicios, no se ha acomodado hasta el presente a su nuevo papel en el marco del Estado autonómico.

\section{La relación con los ciudadanos}

En cuanto a su relación con los ciudadanos, puede decirse de forma sintética que, a pesar de la evidente mejora de las prestaciones públicas, los ciudadanos siguen viendo a la Administración dificilmente accesible en su relación cotidiana, excesivamente compleja y lenta en los procedimientos de actuación y sin posibilidad de que su opinión y sus quejas se escuchen, a no ser por la vía formalizada de los recursos administrativos.

La opinión sobre el mal funcionamiento de los servicios, que como criterio general tienen asumido los ciudadanos, hace que cuando se relacionan con servicios concretos de los que obtienen satisfactoria respuesta a sus necesidades, 
lo consideren como una excepción a la regla general, como han puesto de manifiesto los estudios y encuestas del CIS.

\section{La integración en la U.E. y la economia nacional}

Por último, pero no menos importante, la incorporación de España a la Comunidad Europea supuso la pérdida de determinadas funciones de la Administración del Estado y la asunción de otras nuevas, así como la necesidad de establecer cauces de acruación y estructuras que posibiliten al Estado una adecuada participación en el proceso de formación y ejecución de la voluntad comunitaria. Todo ello requería un nuevo enfoque de sus tareas, que no tuvo adecuado reflejo organizativo. Además, el programa de convergencia hacia la moneda única obliga al Estado espanol a disminuir el déficit presupuestario de las Administraciones Públicas.

En este marco de referencia, es evidente que la reforma de la AGE pasa por un esfuerzo de ahorro del gasto público, comportándose con mayor eficiencia que hasta el presente y mejorando o manteniendo, simultáneamente, la calidad de los servicios que presta a ciudadanos y empresas. Tales objetivos inciden además fuertemente en la productividad del sistema económico español de cara a la unión económica, puesto que muchos de los servicios públicos que ofrece la Administración son inputs para la producción de bienes en el sector privado, como es el caso de las infraestructuras. Además, otros servicios públicos como la educación o la sanidad, que son servicios finales para el consumidor, afectan al factor trabajo y en último término a la productividad del sistema económico nacional.

En conclusión, las exigencias en cuanto a una eficaz y eficiente utilización de los recursos públicos en el contexto económico y social en el que se desenvuelve la AGE se caracterizan por dos notas: en primer lugar, por una presión social creciente sobre la calidad de los servicios públicos, consecuencia del incremento de la presión fiscal a partir de la década de los 80 y de la mejora de la calidad de los bienes y servicios proporcionados por el sector privado, lo que provoca que cada vez más los ciudadanos contribuyentes demanden de la Administración estándares de calidad similares en los servicios públicos. En segundo lugar, la necesidad de una adecuada coordinación y colaboración con las otras Administraciones territoriales para lograr la mejor utilización de los recursos con los que cuentan el conjunto de las Administraciones Públicas.

\section{Propuestas para adecuar la AGE a su nuevo papel}

\section{Programa de reforma}

La estrategia del Gobierno surgido de las últimas elecciones, para adecuar la AGE a las nuevas necesidades, aconsejó remitir rápidamente al Congreso un proyecto de Ley, la LOFAGE, que estableciera el marco de la reforma. Una vez aprobada la Ley por las Cortes, el Gobierno decidió poner en marcha inmediatamente un programa de actuación en materia de Administración periférica y, a medio plazo (máximo de dos años), otro programa referido a los Organismos Públicos. Por lo que atañe a los Ministerios, los Reales Decretos de reorganización de 1996 se apoyaban ya, en cierta medida, en los criterios del proyecto de la LOFAGE, en particular en cuanto al enfoque de los servicios comunes

Apuntaré ahora los rasgos generales del modelo y posteriormente las actuaciones en curso para su puesta en práctica.

\section{Modelo de la LOFAGE}

Un modelo de organización satisfactorio debía establecer las vías de solución de los problemas más relevantes de la Administración General del Estado. Además, las soluciones ofrecidas han de ser duraderas, para asegurar a la Administración un marco cierto y estable de funcionamiento que no necesite ser cambiado según la coyuntura y que encauce sin tensiones los procesos de transformación que la Administración debe afrontar.

En este escenario, el modelo diseñado por la LOFAGE reúne las siguientes características:

Constituye un marco juridico regulador de la organización y el funcionamiento, que asegura el sometimiento de estas cuestiones a reglas incardinadas en el ordenamiento jurídico público, de obligada aplicación en el ejercicio de la potestad organizatoria y en la actuación de los aparatos administrativos.

No busca un modelo organizativo unitario y cerrado, imposible de aplicar a toda la AGE y, menos aún, contener las exigencias de cambio que puedan plantearse en el futuro. Por el contrario, abre la posibilidad de soluciones organizativas diversas (por ejemplo, Ministerios con o sin Secretarías de Estado o Secretarías generales, con un tipo u otro de Organismos Públicos) según la naturaleza de las actividades a desarrollar, pero asegurando la coherencia de di- 
chas soluciones organizativas respecto de los criterios básicos de configuración y funcionamiento (por ejemplo, la relación entre las funciones ministeriales y las propias de sus Organismos Públicos)

Contempla toda la Administración General del Estado: central, periférica, institucional y exterior, con objeto de dotarla de un marco común para el funcionamiento de los servicios públicos estatales.

Examinaré a continuación, con brevedad, los rasgos de dicho modelo:

\section{a) Principios de funcionamiento de la Administración del Estado que incorpora el modelo}

Orientación de la Administración hacia la eficacia y la eficiencia, haciendo énfasis en la finalidad de prestar unos servicios públicos que establezcan estándares de calidad, definiendo las funciones con un enfoque finalista hacia el cumplimiento de objetivos, y promoviendo el empleo de técnicas gerenciales de actuación.

Deslinde de órganos superiores y directivos. Autonomía y responsabilización de los órganos directivos, facilitando la desconcentración/delegación de la gestión de los medios en los responsables de los órganos directivos. Implantación efectiva de sistemas de control que permitan una evaluación real del funcionamiento de los aparatos y servicios públicos, profundizando en el control de eficacia, cuya finalidad es "comprobar el grado de cumplimiento de los objetivos y la adecuada utilización de los recursos asignadosn.

Profesionalización de la Administración y establecimiento de la responsabilidad personal y directa del directivo por la gestión, la evaluación por el superior con arreglo a criterios de competencia y profesionalidad, en el marco de libre nombramiento y cese de estos puestos, y la exigencia de que los titulares de los órganos directivos (salvo Secretarios generales o, excepcionalmente, Directores generales de determinados sectores de actividad) sean ocupados por funcionarios públicos con titulación superior.

\section{b) Criterios organizativos}

El encaje de los distintos tipos organizativos se basa en atribuir a los Ministerios las funciones de la AGE para luego desgajar algunas de ellas en favor de Organismos Autónomos o Entidades Públicas Empresariales; establecer dos tipos organizativos, simplificando la situación actual, y asignar a cada uno de ellos determinados cometidos con su correspondiente modelo organizativo y funcional, dentro de unos márgenes de flexibilidad.

\section{Órganos ministeriales}

Se rigen por el principio de distribución funcional de competencias. Dentro de este esquema, la LOFAGE potencia la figura del Ministro como responsable político máximo de su Ministerio, reforzando su capacidad de decisión sobre la organización y los medios de él dependientes. Al mismo tiempo, se amplían las posibilidades del Ministro de delegar competencias de gestión en los directivos del Ministerio.

Configuración de la estructura básica de los Ministerios sobre las Subsecretarías, Secretarías Generales Técnicas, Direcciones Generales y Subdirecciones Generales como órganos que, en todo caso, deben existir. Las Secretarías de Estado y, excepcionalmente, las Secretarías Generales se crearán para dirigir un sector de actividad del Ministerio, cuando ello sea conveniente.

Rediseño de los servicios comunes para enfocar su actividad desde la óptica de apoyo a las unidades de linea. Los servicios comunes deben, en todo caso, desarrollar funciones estratégicas en relación con los recursos humanos materiales, y de información y servir de apoyo a las funciones del Ministro como responsable máximo del control de eficacia de la gestión del Ministerio.

\section{Criterios de organización de los órganos territoriales de la AGE}

El modelo de la LOFAGE incluye la Administración periférica (tradicionalmente regulada por Decreto) para dar un tratamiento completo y coherente de la AGE, cuyas innovaciones más significativas son:

Reforzamiento de la figura del Delegado del Gobierno en su dimensión política: representante territorial del Gobierno y responsable de la dirección de los servicios de la $A G E$, lo que implica la relación con los máximos responsables ministeriales, la formulación de propuestas sobre los planes de actuación de los Ministerios y su incidencia en el territorio, y la evaluación del funcionamiento de los servicios públicos estatales en el ámbito de la Comunidad Autónoma. Además, se sitúa como máximo responsable territorial de la AGE en la cadena de mando de las Fuerzas y Cuerpos de seguridad del Estado.

Supresión de los Gobernadores civiles y creación de la figura de los Subdelegados del Gobierno: profesionales funcionarios que apoyan al Delegado, que es su superior jerárquico y quien los nombra. El Subdelegado es, pues, un colaborador del Delegado, lo que tiene reflejo en las funciones que se le atribuyen y en el modo de desarrollarlas que establece la LOFAGE. Ello permite, además, 
que el Delegado del Gobiemo pueda delegarle competencias en todo aquello que facilite la gestión, sin riesgo de interferencias.

Integración progresiva pero plena de servicios ministeriales en las Delegaciones y Subdelegaciones del Gobiemo, reestructuración de éstas y dependencia orgánica del MAP, salvo respecto de los servicios que cumplan los requisitos fijados por la LOFAGE: gran volumen de gestión o singularidad de sus funciones. En consecuencia, la organización territorial de la AGE se rige por el principio de gestión integrada.

Simplificación de la estructura territorial de la AGE para evitar duplicidades innecesarias con los correspondientes servicios de las Comunidades Autónomas, con la supresión de direcciones territoriales o provinciales de los Ministerios afectados.

Dirección y supervisión de los servicios no integrados y especial colaboración que éstos deben prestar al Delegado y Subdelegado.

\section{Organismos Públicos}

Tienen carácter instrumental respecto de la naturaleza de la actividad a desarrollar. Cuando dicha actividad reúna las características precisas, y sólo entonces, se crearán Organismos Públicos, por lo que la valoración previa a su creación de su Plan Inicial de Actuación y el proyecto de Estatutos por parte del Gobierno, previsto por la LOFAGE, adquiere particular relevancia.

Las principales innovaciones son las siguientes:

Reducción de la actual multiplicidad de figuras a dos, cuyo criterio diferencial básico estriba en que los Organismos Autónomos realizan actividades (ejercicio de funciones, prestación de servicios) que no son predominantemente económicas y se financian esencialmente con transferencias de los Presupuestos del Estado. Por su parte, las Entidades Públicas Empresariales actuarán mediante contraprestación y deberán generar ingresos con el objetivo de cubrir, al menos, los costes de los bienes que producen o los servicios que prestan, sin percibir consignaciones presupuestarias o transferencias, salvo excepción.

Los Organismos Autónomos, habida cuenta el tipo de actividad que desarrollan, han de regirse plenamente por el Derecho público. Las Entidades Públicas Empresariales, por el contrario, actúan de acuerdo con el ordenamiento privado (de forma similar a los actuales Entes del artículo 6.1. b) TRLGP), pero les será de aplicación el Derecho público en cuanto a los aspectos básicos de organización, for- mación de la voluntad de sus órganos, presupuestación, contabilidad, o al ejercicio de potestades públicas. En ambos casos, los procesos de creación de este tipo de entes han de vincularse, como decíamos, a que se comprometan a realizar una gestión más eficaz y eficiente que bajo una estructura cèntralizada, fijando objetivos concretos de mejora.

\section{Principales actuaciones en aplicación de la LOFAGE}

\section{Reforma de la Administración periférica}

Parece que hay acuerdo en señalar que el aspecto más innovador de los contenidos en la LOFAGE es el relativo a la reforma de la Administración periférica de la Administración General del Estado. En realidad, la novedad, además de en algunos aspectos de la regulación, donde verdaderamente adquiere mayor relieve es en su aplicación, es decir, en la voluntad de llevar a cabo esa reforma.

La desaparición de los Gobernadores civiles, cuya importancia no hay que ocultar, ha podido convertirse exageradảmente en la representación de la reforma de la Administración periférica. Se puede decir que exageradamenten, ya que sólo es una entre el conjunto de medidas que se van a adoptar en este terreno y que han quedado en cierto modo ocultas tras la desaparición de los Gobernadores, que ha resultado, quizás, la medida más espectacular.

Como consecuencia de la desaparición de los Gobemadores civiles se crea una figura que llevará a cabo en el ámbito provincial las funciones relativas a los servicios de la Administración General del Estado, el Subdelegado del Gobierno en la provincia.

Como consecuencia de todo lo anterior, se ha aprobado y ha entrado en vigor el Real Decreto 617/1997, de 25 de abril, de Subdelegados del Gobierno, y en aplicación del mismo el pasado 5 de junio los Gobernadores civiles fueron cesados por el Consejo de Ministros y se nombró a los Subdelegados del Gobierno.

\section{Ia integración de servicios periféricos}

A medio plazo, la integración de los servicios periféricos ministeriales será percibida como la verdadera reforma de la Administración periférica. El Consejo de Ministros aprobó el plan presentado por el Ministro de Administraciones 
Públicas. Este plan que huye de posturas maximalistas para poner en práctica las previsiones de la LOFAGE prevé, en una primera fase, la integración de servicios periféricos de los Ministerios de Agricultura, Pesca y Alimentación, Educación y Cultura, Fomento, Industria y Energía, Sanidad y Consumo, y Trabajo y Asuntos Sociales.

En una fase posterior se procederá a la integración de otro conjunto de servicios periféricos, revestidos de una mayor complejidad técnica, a la que se hará frente una vez que se haya evaluado el resultado de esta primera fase y validados o corregidos los distintos aspectos de la misma.

La primera fase de la integración, que será plena, según nuestros proyectos, a partir de enero de 1998, significará que los efectivos de los servicios integrados, tanto humanos como materiales, pasen a depender de la correspondiente Delegación del Gobierno a través de la relación de puestos de trabajo y del presupuesto, y ello implica, lógicamente, la realización de las correspondientes transferencias patrimoniales o presupuestarias. En cualquier caso, nos hemos esforzado en encontrar fórmulas que no supongan un desapoderamiento de los Ministerios con respecto a sus estructuras periféricas, ya que determinados aspectos técnicos o de política de recursos humanos aconsejaban mantener vínculos que, en ningún caso, cuestionarán el perfeccionamiento de la integración.

\section{La adecuación de los Organismos Públicos a la LOFAGE}

En estos momentos están trabajando conjuntamente los Ministerios de Administraciones Públicas y de Economía y Hacienda en la adecuación de los actuales Organismos Públicos a lo establecido en la LOFAGE. Esta adecuación consiste en integrarlos en uno de los dos tipos definidos por la misma. En términos generales, los Organismos Autónomos de carácter administrativo no deben tener problemas para pasar a ser Organismos Autónomos-LOFAGE ni los Entes del art. 6.1.b) LGP

\footnotetext{
$\mathrm{N}^{\circ}$ de ordenadores

Valor del parque informático (M. ptas. corrientes de cada año)

N. ${ }^{\circ}$ de terminales de pantalla-teclado por cada 100 empleados
}

En 1975 el ordenador de la Administración que disponía de una memoria central mayor era el del Centro de Proceso de Datos del Ministerio de Obras Públicas, con una memoria de 1.920 kilocaracteres, que hoy es superada ampliamente por los ordenadores personales de sobremesa. Del mismo modo, el ordena- para acomodarse al tipo de Entidad Pública Empresarial. Más dificultades puede plantear la reubicación de los actuales Organismos Autónomos comerciales que no reúnen las condiciones precisas para ser Entidades Públicas Empresariales pero cuyas operaciones comerciales tienen cierto relieve.

La Ley establece un plazo de dos años para llevar a cabo esta adecuación, manteniendo el actual régimen de cada Organismo hasta que se produzca su acomodación a la LOFAGE. Ello va a permitir estudiar detenidamente las posibles consecuencias de las decisiones que se adopten y que el Ministerio de Economia y Hacienda defina el régimen que se incorporará en la futura Ley General presupuestaria, imprescindible puesto que la LOFAGE se remite a esta Ley en diversos aspectos esenciales para el funcionamiento de los Organismos Públicos.

\section{Particular consideración de la reforma de la AGE en los aspectos relacionados con las tecnologías de la información}

\section{El punto de partida}

Para dar una primera idea de la evolución en este sector desde la situación preconstitucional a nuestros días, en términos cuantitativos, podemos partir de las siguientes cifras referentes a la que hoy conocemos como Administración General del Estado:

$\begin{array}{rc}1975 & 1996 \\ 92 & 151.791 \\ 3.068 & 59.971 \\ 0,1 \% & 24 \%\end{array}$

dor con un mayor volumen de capacidad en disco era el de las Mutualidades Laborales del Ministerio de Trabajo con 3 gigacaracteres de almacenamiento. Ahora un ordenador de sobremesa tiene no menos de 1 gigacarácter. 


\section{Las etapas de la transformación}

Así, en una primera etapa, cuya finalización podemos situar a comienzos de la década de los 80 , las aplicaciones de las $\mathrm{TI}$, se orientan principalmente a la mejora de la gestión administrativa, en el sentido de una mayor productividad: automatización de procedimientos que implican el manejo de elevados volúmenes de información repetitiva. Por el contrario, no aparecen aplicaciones dirigidas a innovar o modernizar la propia Administración, atendiendo principalmente a la prestación de los servicios públicos, en línea con los objetivos de un plan estratégico global (no específicamente de T1) de alcance ministerial o general.

Una segunda etapa, que llegaría hasta principios de los anos 90 , se caracteriza por un planteamiento de las tecnologías como instrumento. Desde un punto de vista general, se inicia un cambio de tendencia, expresivo de una toma de conciencia del papel que los sistemas y tecnologias de la información pueden desempeñar en el proceso de modernización administrativa, aunque por lo general, la aproximación a la planificación y el desarrollo de sistemas de información se caracteriza por enfoques todavía esencialmente artesanales, sin el soporte que pudiera aportar un adecuado marco metodológico de ingeniería del software.

Aparecen nuevos proyectos de carácter estratégico. Entre ellos cabe destacar los sistemas de soporte a la contabilidad y el presupuesto, el proyecto ATENEA, orientado a la introducción de la informática en las enseñanzas no universitarias, y el proyecto INFORIUS, para atender a las necesidades informáticas de la Administración de Justicia. Todos estos proyectos son plurianuales, y a cada uno de ellos se han dedicado recursos por importe del orden de varios miles de millones de Ptas.

La tercera etapa en el modelo que venimos utilizando para representar la evolución del uso de las TI en la Administración del Estado en España puede considerarse iniciada con la puesta en marcha bajo el impulso del Ministerio para las Administraciones Públicas del Programa de Modernización de la Administración del Estado.

El Programa, cuyos trabajos preparatorios se iniciaron en 1990 con el documento del MAP "Reflexiones sobre la Modernización de la Administración del Estado propone: Mejorar la planificación y la toma de decisiones, mejorar la prestación del servicio público en relación a los ciudadanos y abrir el camino a la desconcentración de las estructuras organizativas.

Si vamos más allá de los principios programáticos y tratamos de examinar el marco legislativo, nos encontramos que la Ley 30/92, de 26 de noviembre, de Régimen Jurídico de las Administraciones Públicas y del Procedimiento Administrativo
Común, introduce en su artículo 45 un conjunto de disposiciones orientadas a lo que la propia Ley denomina sla tecnificación y modernización de la actuación administrativar. Puede senalarse aquí que el articulo 45 de la LRJ-PAC constituye un paso adelante esencial en la remoción de barreras legales que dificultaban o impedian el despliegue de las TI en las Administraciones que, de acuerdo con esta norma, pueden utilizarse no sólo para la gestión interna, sino también para las relaciones con los ciudadanos.

\section{La situación actual}

Por último, dentro de este punto dedicado a las TI ¿dónde nos encontramos ahora?, ¿cómo entendemos que debe abordarse el futuro? Se trata de avanzar hacia la Administración electrónica, entendida ésta como la posibilidad de que los ciudadanos accedan a los servicios administrativos de manera electrónica, 24 horas al día, 7 días a la semana, para la obtención de información. Además, la posibilidad de efectuar trámites de manera electrónica, con los ciudadanos, con otros órganos o Administraciones, con las empresas. También, la reducción y sustitución del papeleo, gracias a la extensión del correo electrónico.

En este contexto cobra especial relevancia el Proyecto "Ventanilla Única" puesto en marcha con el Acuerdo de 4-4-1997 del Consejo de Ministros para la progresiva implantación de un sistema intercomunicado de registros entre la Administración General del Estado, las Administraciones de las Comunidades Autónomas y las entidades que integran la Administración Local. El Acuerdo contempla la .Ventanilla Única como un conjunto de medidas e instrumentos de colaboración interadministrativa que permitan un proceso coordinado de implantación de un sistema intercomunidado de los registros administrativos, el intercambio de las bases de datos e instrumentos de información y atención al ciudadano de las respectivas Administraciones y la simplificación e integración de los trámites y procedimientos administrativos en que participen las administraciones intervinientes.

En estos momentos nos encontramos trabajando en la puesta en marcha del proyecto, con la colaboración de las Administraciones de las Comunidades Autónomas y de la FEMP. Por su propia naturaleza se trata de un proyecto a largo plazo, pero de implantación progresiva, de modo que a lo largo del próximo año podamos ver aplicaciones prácticas en ámbitos administrativos específicos. En ellas han de jugar un papel fundamental las tecnologías de correo electrónico y del mundo Internet, así como las que proporcionan servicios de seguridad orientados al procedimiento (firma electrónica, estampación de fecha, etc.). 
- Directora General de Organización Administrativa. Ministerio de Administraciones Públicas. 